\section{Mortes perinatais e avaliação da assistência ao parto em maternidades do Sistema Único de Saúde em Belo Horizonte, Minas Gerais, Brasil, 1999}

\author{
Perinatal deaths and childbirth healthcare \\ evaluation in maternity hospitals of the Brazilian \\ Unified Health System in Belo Horizonte, \\ Minas Gerais, Brazil, 1999
}

\author{
Sônia Lansky 1,2 \\ Elisabeth França 2 \\ Cibele Comini César 3 \\ Luiz Costa Monteiro Neto 2 \\ Maria do Carmo Leal 4
}

\section{Introdução}

No Brasil são poucos os estudos publicados sobre a mortalidade perinatal, muito embora seja um importante indicador da assistência obstétrica e neonatal e do nível sócio-econômico da população. Os dados disponíveis no país referem-se a realidades locais e mostram que os nossos índices estão muito além dos aceitáveis, duas a três vezes maiores quando comparados com taxas de países desenvolvidos e mesmo com alguns países em desenvolvimento 1,2 As afecções perinatais se constituem não apenas como a primeira causa de mortalidade neonatal no país, mas também das mortes que ocorrem ao longo do primeiro ano de vida; são responsáveis ainda por $48,5 \%$ das mortes de crianças menores de cinco anos 3 .

A concentração dos óbitos neonatais nas primeiras horas de vida e a freqüente ocorrência de óbitos fetais no final da gestação e durante o trabalho de parto evidenciam a estreita relação entre estas mortes e a qualidade da assistência nos serviços de saúde 4,5 . Ainda que os fatores de risco para a morte perinatal se articulem de maneira complexa 6 , é necessário agregar àqueles fatores mais tradicionais e reconhecidos na literatura - como as condições sócio-econômicas, condições maternas e do recém-nascido - informações relativas aos serviços de saúde. Os altos e persistentes índices de mortalidade neonatal e perinatal deman- 
dam a realização de estudos de avaliação da qualidade da assistência obstétrica e neonatal prestada nos serviços.

Uma parcela importante dos óbitos perinatais ocorre em crianças com peso ao nascer acima de 1.500 g e sem malformação congênita grave e ou letal, podendo, portanto, ser considerada prevenível pelo acesso oportuno a serviços qualificados de atenção pré-natal, ao parto e ao recém-nascido $1,4,7,8,9,10,11$. Óbitos nesta faixa de peso, sobretudo as mortes decorrentes de asfixia intraparto, deveriam ser considerados eventos sentinelas por todos os serviços que prestam assistência à gestante e ao recémnascido, por apresentarem grande potencial de redução pelas ações diretas de saúde 11,12,13 .

Nos países desenvolvidos houve declínio significativo na taxa de mortalidade perinatal, principalmente de mortes intraparto 14 , o que não foi observado no Brasil 15. A asfixia é apontada como uma das principais causas de mortalidade perinatal em países em desenvolvimento 4,11, em contraposição ao contexto atual dos países desenvolvidos, onde predominam a prematuridade extrema e a malformação congênita 16. É ainda a principal causa de óbito nos locais onde a taxa de mortalidade fetal é elevada 11 .

No Brasil, poucos estudos têm enfatizado a contribuição dos aspectos relativos à assistência em maternidades na definição do padrão da mortalidade perinatal, numa realidade em que a grande maioria dos partos é hospitalar $(91,5 \%)$ e realizada por médicos $(77,6 \%)$, diferente de muitos países em desenvolvimento, onde predominam partos domiciliares 17. Alguns estudos apontaram falhas na estrutura da assistência perinatal no Brasil 18,19, outros, realizados em Belo Horizonte, revelaram condições inadequadas para o atendimento perinatal no que diz respeito à estrutura hospitalar e perfil dos nascimentos nos estabelecimentos de saúde 20,21. Persiste, no entanto, a necessidade de realização de estudos de avaliação do processo de assistência obstétrica e neonatal prestada nos serviços, enfoque deste estudo.

O termo "processo" se constitui por tudo aquilo que os médicos e demais profissionais realizam na assistência à saúde dos pacientes, bem como a habilidade com que a disponibilizam. Refere-se também à proficiência técnica e à interação com os pacientes 22,23. A utilização dos indicadores de processo e de resultados da assistência (o óbito perinatal), em conjunto com a avaliação da estrutura dos serviços, permitem uma avaliação mais ampla da assistência prestada nessa área. A avaliação da assistência perinatal, particularmente a assis- tência ao parto, faz-se necessária, pois há poucas informações sobre o atendimento ao parto e aos recém-nascidos no país. A constatação de altos coeficientes de mortalidade perinatal apesar da distribuição relativamente adequada do peso ao nascer - sugere que sua qualidade é baixa 2 .

O objetivo deste estudo é analisar a associação entre a morte perinatal e variáveis relacionadas com o processo de assistência hospitalar ao parto, embora reconhecendo a profunda influência dos fatores sócio-econômicos e da organização da rede assistencial de saúde como a regionalização, integralidade e hierarquização da assistência para a mortalidade perinatal. Enfoca especificamente ações de saúde relativas ao processo de atendimento nas maternidades que são passíveis de intervenção direta, com um grande potencial de produzir modificações importantes na mortalidade fetal, neonatal e infantil.

\section{Material e métodos}

\section{Delineamento do estudo}

Trata-se de estudo caso-controle de base populacional dos óbitos perinatais (casos) e dos nascimentos que sobreviveram ao período neonatal precoce (controles), ocorridos em 15 maternidades do Sistema Único de Saúde (SUS) em Belo Horizonte, Minas Gerais, Brasil, entre janeiro e setembro de 1999.

\section{Fontes de dados}

As informações dos casos e controles foram coletadas dos prontuários hospitalares das gestantes e recém-nascidos e laudos de necropsia, em formulário padrão. Algumas informações não disponíveis nos prontuários, como escolaridade materna e paridade, foram obtidas com a utilização dos dados do Sistema de Informações sobre Mortalidade (SIM) e do Sistema de Informações de Nascidos Vivos (SINASC), por meio do relacionamento dos bancos.

\section{Seleção da amostra}

Para a seleção dos casos foram inicialmente considerados todos os óbitos fetais e neonatais precoces ocorridos no período em maternidades do SUS ( $\mathrm{n}=479$ ), a partir de investigação dos óbitos perinatais de 1999 1. Os controles foram selecionados com base em amostra aleatória $(\mathrm{n}=1.077)$ dos 19.916 nascimentos ocorridos no período nos hospitais do SUS, em es- 
tudo realizado pela Secretaria Municipal de Saúde de Belo Horizonte (SMSA-BH): Qualimat 24 . Foram utilizados critérios fixos para o cálculo da amostra dos controles, estratificada por hospital, sem alocação proporcional: prevalência do evento (baixa qualidade da assistência) de $30 \% ; \alpha=0,05 ; \beta=10 \%$; precisão de $20 \%$, resultando em 1.077 nascimentos. Houve perda de sete casos e oito controles por falta de informação sobre o motivo de internação da gestante, além de outros 11 casos e seis controles por falta de registro de data ou hora de admissão na maternidade ou do parto.

Foram excluídas do estudo as crianças de gestantes não residentes em Belo Horizonte e as nascidas com menos que $500 \mathrm{~g}$ de peso ou 22 semanas de idade gestacional 25 . Somente foram incluídas as gestantes com gravidez única, admitidas em trabalho de parto nas maternidades com fetos vivos, e que permaneceram pelo menos uma hora em trabalho de parto no hospital. A amostra final do estudo foi constituída por 118 óbitos perinatais e 492 controles. Estima-se que essa amostra tenha um poder de $80 \%$ para detectar um odds ratio (OR) de pelo menos 1,85 , para uma prevalência de exposição entre os controles de $30 \%$ e um nível de significância de $5 \%$ (teste dos dois lados).

Realizou-se estudo de confiabilidade para avaliação da qualidade dos dados coletados, dado que os controles foram selecionados a partir de banco de dados secundário (Qualimat). Utilizou-se amostra aleatória de 43 (20\%) dos prontuários em 6 dos 15 hospitais participantes do estudo, selecionados pelo maior volume de atendimentos e/ou maior número de perdas de prontuários, representando $12 \%$ da amostra inicial. Verificou-se concordância boa, com índice kappa entre 0,6 e 0,9 para as variáveis de interesse deste estudo. Outro estudo comparando o SINASC e o banco Qualimat também demonstrou boa concordância para as principais variáveis utilizadas neste trabalho 26 .

\section{Variáveis estudadas}

As principais variáveis de exposição, de acordo com o propósito do estudo de avaliação do processo de assistência à gestante em trabalho de parto nas maternidades foram: (1) uso de partograma para acompanhamento do trabalho de parto; (2) taxa de avaliação materna e fetal durante o trabalho de parto (total de avaliações realizadas dividido pelo número de horas entre a admissão da gestante no hospital e o parto). Considerou-se que o partograma foi utilizado quando havia registro dos dados de evolução do trabalho de parto (dinâmica uterina com duração e/ou freqüência das contrações e/ou dilatação do colo e/ou altura e ausculta fetal e/ou condição da bolsa das águas) em qualquer tabela ou gráfico, já que a grande maioria dos serviços de saúde não utilizava a curva de acompanhamento da dilatação cervical em gráfico, conforme recomendado 27,28. A taxa de avaliação materna foi considerada inadequada quando se realizou menos de uma avaliação por hora.

Os possíveis fatores de confusão selecionados foram: (1) instrução materna (menos de 8, 8 e mais anos de estudo); (2) idade materna (menor de 20 anos, de 20 a 34 e acima de 35 anos); (3) idade gestacional (menos de 37, 37 e mais semanas); (4) realização de pré-natal (sim, não); (5) sexo da criança (masculino, feminino); (6) peso ao nascer (menor de $2.500 \mathrm{~g}, 2.500 \mathrm{~g}$ e mais); (7) momento do nascimento (parto diurno/em dia útil, parto ocorrido à noite/em dias de feriado/final de semana); (8) tipo de parto (vaginal, cesárea); (9) presença de doenças maiores na gravidez (hipertensão/DHEG, diabetes/diabetes gestacional, hemorragia e infecção do trato urinário); (10) presença de doenças menores na gravidez (anemia, epilepsia, DST, ou outras); (11) presença de doenças do recém-nascido (infecção, distúrbios metabólicos e malformação congênita grave/letal); (12) Apgar no quinto minuto $(<7 ; \geq 7)$; (13) assistência de pediatra na sala de parto ( $\mathrm{sim}$, não); (14) tipo de hospital (hospital tipo 1: sem unidade neonatal intermediária ou intensiva; hospital tipo 2: com unidade de cuidado neonatal intermediário ou semi-intensivo; hospital tipo 3: com unidade de cuidado neonatal intensivo), tomando-se como referência a classificação de Costa et al. 21 e da Comissão Perinatal da SMSA-BH 29.

O motivo da internação, uso de corticosteróide, local de permanência do recém-nascido e a categorização do óbito segundo os critérios de evitabilidade da classificação de Wigglesworth modificada 13,30,31 foram utilizados somente na análise descritiva.

\section{Análise estatística}

Mediu-se a associação entre cada variável considerada e a morte perinatal por meio do OR, com intervalo de confiança de 95\% (IC95\%). Análise univariada foi realizada para variáveis selecionadas, comparando casos e controles, sendo excluídos os registros sem informação para as estimativas de OR. Em toda a análise estatística os controles foram ponderados pelo inverso da probabilidade de inclusão na amostra para corrigir a alocação não proporcional 
da amostragem original dos controles. Análise de regressão logística múltipla não condicional foi realizada para obtenção de estimativas independentes para o risco de morte perinatal, com inclusão das variáveis estatisticamente significativas na análise univariada $(\mathrm{p}<0,25)$. Optou-se pela não inclusão da variável idade gestacional no modelo, devido à melhor qualidade da variável peso ao nascer e à forte correlação entre essas duas variáveis 14 . Foram construídos três modelos de regressão logística múltipla, um para cada variável de exposição principal consideradas neste estudo: utilização de partograma durante o parto, taxa de avaliação materna e taxa de avaliação fetal por hora durante o trabalho de parto. Duas variáveis foram excluídas dos modelos de regressão: Apgar no quinto minuto, por se considerar como evento intermediário na cadeia causal do óbito; e a assistência pediátrica na sala de parto, por se tratar de situação restrita aos 86 nascidos vivos no grupo de casos. Cada variável foi avaliada segundo o teste de Wald e o OR, com IC95\%, estimado a partir do coeficiente b e seu respectivo erro-padrão. Para o relacionamento dos bancos e análises utilizaram-se os pacotes estatísticos Stata 8.0 (Stata Corporation, College Station, Estados Unidos) e SPSS 10.0 (SPSS Inc., Chicago, Estados Unidos).

A pesquisa foi aprovada pelo Comitê de Ética em Pesquisa da Universidade Federal de Minas Gerais e obteve-se o consentimento dos hospitais para a sua participação no estudo.

\section{Resultados}

Os resultados da análise univariada são apresentados nas Tabelas 1 e 2 . A idade materna (média de 24,5 variando entre14 e 47 anos) e escolaridade materna ( $77 \%$ da população com menos de oito anos de estudo) não apresentaram associação com o óbito perinatal. O subregistro para a variável paridade da mãe foi elevado ( $80,5 \%$ dos casos e $27,6 \%$ dos controles), impossibilitando a sua utilização na análise de risco para o óbito perinatal. A idade gestacional (média de 37,3 semanas, variando entre 17 e 43 semanas de gestação) também apresentou percentual elevado de registros ignorados, 15,2\% dos casos e 17,9\% dos controles. Grande parte dos óbitos ocorreu em crianças prematuras (55,4\% dos casos e $6,5 \%$ dos controles, $\mathrm{OR}=18,22$; IC95\%: 10,42-31,86) e o baixo peso ao nascer $(<2.500 \mathrm{~g})$ estava presente em $19,2 \%(\mathrm{n}=120)$ dos nascimentos, sendo $61 \%(n=72)$ entre os casos e $8,1 \%(n=40)$ dos controles, com forte associação com o óbito perinatal $(\mathrm{OR}=17,60$;
IC95\%: 10,55-29,34). Apenas 0,6\% dos controles tinha muito baixo peso ao nascer $(<1.500 \mathrm{~g})$, em contraste com $40,5 \%$ dos casos, embora uma proporção elevada de óbitos $(37,9 \%)$ tenha ocorrido em crianças com peso acima de 2.500g.

A ausência de informação sobre o pré-natal foi elevada entre os óbitos (33\%) e a não realização deste apresentou-se como fator de risco importante para o óbito perinatal $(\mathrm{OR}=12,99$; IC95\%: 6,27-26,92). “Doenças maiores” e “doenças menores” na gravidez foram mais prevalentes entre os casos (OR = 5,29; IC95\%: 2,80-10,02 e OR = 7,26; IC95\%: 4,13-12,74, respectivamente). A maioria dos nascimentos e óbitos ocorreu em hospitais tipo $1(56,7 \%)$ e não houve associação entre o óbito perinatal e a categoria do hospital na análise univariada. O partograma foi menos utilizado entre os casos $(\mathrm{OR}=$ 2,29; IC95\%: 1,49-3,53), assim como o acompanhamento fetal inadequado durante o trabalho de parto (OR = 1,91; IC95\%: 1,13-3,21), tendo sido realizada menos de uma avaliação por hora em $82,2 \%$ dos casos e $73,4 \%$ dos controles. De maneira semelhante, $79,7 \%(n=486)$ dos casos e controles tiveram avaliação materna inadequada (menos uma avaliação por hora), sendo 83,9\% ( $\mathrm{n}=99)$ entre os casos e $78,7 \%(\mathrm{n}=$ 387) entre os controles; não houve, porém, associação estatisticamente significativa com o óbito perinatal. A maior parte dos nascimentos ocorreu por via vaginal $(78,8 \%$ dos casos e $78,9 \%$ dos controles) sem associação estatística com o óbito perinatal, o mesmo ocorrendo com o momento do nascimento. $\mathrm{O}$ recém-nascido do sexo masculino representou risco para a morte no período perinatal, como descrito na literatura.

Metade dos nascidos vivos que morreram sofreram asfixia moderada ao nascimento $(\mathrm{OR}=$ 77,30; IC95\%: 30,67-194,82) e um terço tinha peso ao nascer maior que $2.500 \mathrm{~g}$. As crianças que apresentaram "doenças do recém-nascido" não relacionadas com o acompanhamento do trabalho de parto e as crianças que receberam assistência de pediatra na sala de parto tiveram maior risco de óbito perinatal $(\mathrm{OR}=81,05$; IC95\%: 10,59-620,35 e OR = 2,75; IC95\%: 1,16 $6,55)$, neste caso possivelmente por sua condição de maior gravidade. Apenas $0,6 \%(n=4)$ da população em estudo utilizou corticosteróide durante assistência à gestante em trabalho de parto, todos entre os controles, sendo três prematuros. Foram admitidas no período expulsivo do parto $8,4 \%$ das gestantes cujos filhos morreram (casos), em contraste com $0,8 \%$ das gestantes do grupo controle.

Dentre os óbitos, $72,8 \%(\mathrm{n}=86)$ nasceram vivos e $27,1 \%(n=32)$ foram natimortos. Se- 
Tabela 1

Análise univariada entre casos de óbitos perinatais e controles, segundo características selecionadas.

Belo Horizonte, Minas Gerais, Brasil, janeiro-setembro de 1999.

\begin{tabular}{|c|c|c|c|c|c|c|}
\hline \multirow[t]{2}{*}{ Variável } & \multicolumn{2}{|c|}{ Casos } & \multicolumn{2}{|c|}{ Controles } & \multirow[t]{2}{*}{ OR* (IC95\%) } & \multirow[t]{2}{*}{$\mathrm{p}$} \\
\hline & $\mathrm{n}$ & $\%$ & $\mathrm{n}$ & $\%$ & & \\
\hline \multicolumn{7}{|c|}{ Idade materna (anos) } \\
\hline $20-34$ & 66 & 55,93 & 325 & 66,06 & 1 & \\
\hline$<20$ & 33 & 27,37 & 127 & 25,81 & $1,24(0,78-1,99)$ & 0,37 \\
\hline$\geq 35$ & 14 & 11,86 & 37 & 7,52 & $1,92(0,96-3,82)$ & 0,06 \\
\hline Ignorado & 5 & 4,24 & 3 & 0,61 & & \\
\hline Total & 118 & 100,00 & 492 & 100,00 & & \\
\hline \multicolumn{7}{|c|}{ Instrução materna (anos) } \\
\hline$>8$ & 21 & 17,80 & 99 & 20,12 & 1 & \\
\hline$<8$ & 77 & 65,25 & 330 & 67,07 & $1,19(0,69-2,05)$ & 0,53 \\
\hline Ignorado & 20 & 16,95 & 63 & 12,81 & & \\
\hline Total & 118 & 100,00 & 492 & 100,00 & & \\
\hline \multicolumn{7}{|l|}{ Pré-natal } \\
\hline Sim & 59 & 50,03 & 475 & 96,54 & 1 & \\
\hline Não & 20 & 16,95 & 17 & 3,46 & $12,99(6,27-26,92)$ & 0,00 \\
\hline Ignorado & 39 & 33,05 & 0 & 0,00 & & \\
\hline Total & 118 & 100,00 & 492 & 100,00 & & \\
\hline \multicolumn{7}{|c|}{ Idade gestacional (semanas) } \\
\hline$>37$ & 37 & 31,36 & 372 & 75,61 & 1 & \\
\hline$<37$ & 63 & 53,39 & 32 & 6,50 & $18,22(10,42-31,86)$ & 0,00 \\
\hline Ignorada & 18 & 15,25 & 88 & 17,89 & & \\
\hline Total & 118 & 100,00 & 492 & 100,00 & & \\
\hline \multicolumn{7}{|c|}{ Doenças maiores na gravidez } \\
\hline Não & 93 & 78,81 & 471 & 95,73 & 1 & \\
\hline Sim & 25 & 21,19 & 21 & 4,27 & $5,29(2,80-10,02)$ & 0,00 \\
\hline Total & 118 & 100,00 & 492 & 100,00 & & \\
\hline \multicolumn{7}{|c|}{ Doenças menores na gravidez } \\
\hline Não & 78 & 66,10 & 465 & 94,51 & 1 & \\
\hline $\operatorname{Sim}$ & 40 & 33,90 & 27 & 5,49 & $7,26(4,13-12,74)$ & 0,00 \\
\hline Total & 118 & 100,00 & 492 & 100,00 & & \\
\hline \multicolumn{7}{|c|}{ Momento do nascimento } \\
\hline Equipe completa & 47 & 39,83 & 190 & 38,62 & 1 & \\
\hline Equipe reduzida & 71 & 60,17 & 302 & 61,38 & $0,99(0,65-1,50)$ & 0,95 \\
\hline Total & 118 & 100,00 & 492 & 100,00 & & \\
\hline \multicolumn{7}{|l|}{ Tipo de parto } \\
\hline Normal & 93 & 78,81 & 388 & 78,86 & 1 & \\
\hline Cesárea & 25 & 21,19 & 104 & 21,14 & $1,04(0,64-1,71)$ & 0,87 \\
\hline Total & 118 & 100,00 & 492 & 100,00 & & \\
\hline \multicolumn{7}{|l|}{ Sexo da criança } \\
\hline Feminino & 72 & 61,02 & 240 & 48,78 & 1 & \\
\hline Masculino & 44 & 37,29 & 250 & 50,81 & $1,61(1,05-2,47)$ & 0,027 \\
\hline Ignorado & 2 & 1,69 & 2 & 0,41 & & \\
\hline Total & 118 & 100,00 & 492 & 100,00 & & \\
\hline
\end{tabular}

* Odds ratio bruta; excluídos registros ignorados. 
Análise univariada entre casos de óbitos perinatais e controles, segundo características selecionadas.

Belo Horizonte, Minas Gerais, Brasil, janeiro-setembro de 1999.

\begin{tabular}{|c|c|c|c|c|c|c|}
\hline \multirow[t]{2}{*}{ Variável } & \multicolumn{2}{|c|}{ Casos } & \multicolumn{2}{|c|}{ Controles } & \multirow[t]{2}{*}{$\mathrm{OR}^{\star}(\mathrm{IC} 95 \%)$} & \multirow[t]{2}{*}{$\mathrm{p}$} \\
\hline & $\mathrm{n}$ & $\%$ & $\mathrm{n}$ & $\%$ & & \\
\hline \multicolumn{7}{|c|}{ Peso ao nascer (g) } \\
\hline$\geq 2.500$ & 44 & 37,29 & 450 & 91,46 & 1 & \\
\hline$<2.500$ & 72 & 61,02 & 40 & 8,13 & $17,60(10,55-29,34)$ & 0,00 \\
\hline Ignorado & 2 & 1,69 & 2 & 0,41 & & \\
\hline Total & 118 & 100,00 & 492 & 100,00 & & \\
\hline \multicolumn{7}{|c|}{ Apgar 5 o minuto (nascido vivo) } \\
\hline$\geq 7$ & 38 & 44,19 & 440 & 89,43 & 1 & \\
\hline$<7$ & 44 & 51,16 & 7 & 1,42 & $77,30(30,67-194,82)$ & 0,00 \\
\hline Ignorado & 4 & 4,65 & 45 & 9,15 & & \\
\hline Total & 86 & 100,00 & 492 & 100,00 & & \\
\hline \multicolumn{7}{|c|}{$\begin{array}{l}\text { Pediatra na sala de parto } \\
\text { (nascido vivo) }\end{array}$} \\
\hline Sim & 73 & 84,88 & 359 & 72,97 & $2,75(1,16-6,55)$ & 0,02 \\
\hline Não & 6 & 6,98 & 124 & 25,20 & 1 & \\
\hline Ignorado & 7 & 8,14 & 9 & 1,83 & & \\
\hline Total & 86 & 100,00 & 492 & 100,00 & & \\
\hline \multicolumn{7}{|c|}{$\begin{array}{l}\text { Doença do recém-nascido } \\
\text { (nascido vivo) }\end{array}$} \\
\hline Não & 102 & 86,44 & 491 & 99,80 & 1 & \\
\hline Sim & 16 & 13,56 & 1 & 0,20 & $81,05(10,59-620,35)$ & 0,00 \\
\hline Total & 118 & 100,00 & 492 & 100,00 & & \\
\hline \multicolumn{7}{|c|}{ Categoria de hospital } \\
\hline Tipo 3 & 19 & 16,10 & 78 & 15,85 & 1 & \\
\hline Tipo 2 & 28 & 23,73 & 139 & 28,26 & $0,91(0,47-1,74)$ & 0,77 \\
\hline Tipo 1 & 71 & 60,17 & 275 & 55,89 & $0,99(0,56-1,77)$ & 0,99 \\
\hline Total & 118 & 100,00 & 492 & 100,00 & & \\
\hline \multicolumn{7}{|l|}{ Partograma } \\
\hline Sim & 71 & 60,17 & 379 & 77,03 & 1 & \\
\hline Não & 43 & 36,44 & 105 & 21,34 & $2,29(1,49-3,53)$ & 0,00 \\
\hline Ignorado & 4 & 3,39 & 8 & 1,63 & & \\
\hline Total & 118 & 100,00 & 492 & 100,00 & & \\
\hline \multicolumn{7}{|c|}{$\begin{array}{l}\text { Avaliação fetal durante } \\
\text { trabalho de parto (por hora) }\end{array}$} \\
\hline$\geq 1$ & 21 & 17,80 & 131 & 26,63 & 1 & \\
\hline$<1$ & 97 & 82,20 & 361 & 73,37 & $1,91(1,13-3,21)$ & 0,01 \\
\hline Total & 118 & 100,00 & 492 & 100,00 & & \\
\hline \multicolumn{7}{|c|}{$\begin{array}{l}\text { Avaliação materna durante } \\
\text { trabalho de parto (por hora) }\end{array}$} \\
\hline$\geq 1$ & 19 & 16,10 & 105 & 21,34 & 1 & \\
\hline$<1$ & 99 & 83,90 & 387 & 78,66 & $1,37(0,79-2,37)$ & 0,26 \\
\hline Total & 118 & 100,00 & 492 & 100,00 & & \\
\hline
\end{tabular}

* Odds ratio bruta; excluídos registros ignorados. 
gundo a classificação de Wigglesworth, metade das mortes ( $50 \%$; $\mathrm{n}=59$ ) foi atribuída à "asfixia intraparto", ou seja, ocorreu durante o trabalho de parto ou nascimento. Mais da metade das crianças que morreram por asfixia intraparto $(52,5 \% ; \mathrm{n}=31)$ tinha peso ao nascer acima de $2.500 \mathrm{~g}$ e entre essas $32,3 \%(\mathrm{n}=10)$ nasceram mortas, ou seja, o óbito ocorreu intraútero durante o trabalho de parto/nascimento, apesar das gestantes terem permanecido em assistência na maternidade por no mínimo uma hora. Em 84,7\% ( $n=50)$ destes casos a avaliação fetal foi inadequada durante o trabalho de parto. A “imaturidade" respondeu por $33,9 \%$ das causas de óbitos, seguida pela " malformação congênita gravelletal" (9,3\%) e "condições específicas” (6,8\%). Entre as 86 crianças nascidas vivas que evoluíram para o óbito perinatal, cerca de $40 \%(n=37)$ permaneceram em assistência em unidades de baixa complexidade (alojamento conjunto ou berçário comum) e poucas crianças $(5,8 \%)$ foram assistidas em UTI neonatal.

Todas as variáveis estatisticamente significativas na análise univariada foram utilizadas para a construção dos modelos de regressão múltipla (Tabela 3). Apesar de não significativa na análise univariada, a categoria de hospital foi incluída nos modelos de regressão, dado o interesse de se avaliar as variáveis de processo e estrutura relacionadas com a atenção ao parto e o óbito perinatal. Após o controle dos possíveis fatores de confusão, a utilização de partograma permaneceu associada de maneira independente ao óbito perinatal $(\mathrm{OR}=2,76$; IC95\%: 1,21-6,26; $p=0,015)$ e, em todos os modelos de regressão, o hospital tipo 1 passou a apresentar associação com o óbito perinatal. A avaliação fetal durante o trabalho de parto deixou de ser significativa quando analisada em conjunto com as demais variáveis $(\mathrm{OR}=1,75$; IC95\%: 0,61-5,02) e a avaliação materna durante trabalho de parto permaneceu não significativa com o óbito na análise multivariada. Não realização de pré-natal, baixo peso ao nascer, "doenças maiores" e "doenças menores" na gravidez e "doenças do recém-nascido" permaneceram significativas em todos os três modelos construídos. No modelo partograma o sexo masculino ficou no limite de significância $(\mathrm{p}=$ 0,054) e a idade materna acima de 35 anos não foi significativa para o óbito perinatal; por outro lado esta variável se apresentou como risco independente para o óbito perinatal nos demais modelos. Foi testada a interação entre as variáveis hospital tipo 1 e partograma, baixo peso do recém-nascido com avaliação fetal por hora durante o trabalho de parto e baixo peso do recém-nascido com partograma, não sendo encontrada associação estatisticamente significativa.

\section{Discussão}

Os resultados deste estudo indicam pontos importantes de estrangulamento na atenção perinatal, podendo subsidiar o planejamento de medidas necessárias para melhoria da qualidade da assistência nesta área. Por ser um estudo de base populacional, é representativo dos óbitos e nascimentos da população usuária do SUS e até mesmo dos óbitos perinatais do município de forma geral (exceto as mortes anteparto, excluídas deste estudo e os óbitos ocorridos nos hospitais não conveniados ao SUS), dado que dos 826 óbitos perinatais ocorridos e investigados em Belo Horizonte em 1999, 78,2\% ocorreram nas maternidades conveniadas ao SUS 1.

O relacionamento do banco de dados de casos e controles com os bancos do SIM e SINASC possibilitou a busca de informação quando não havia registro nos prontuários médicos, porém persistiu um elevado percentual de informações ignoradas para paridade (em $80,5 \%$ dos casos e $27,6 \%$ dos controles) e realização de prénatal, com $33 \%$ dos casos sem registro desta informação. Ainda assim, a não realização de pré-natal (considerando-se apenas se este foi realizado ou não, sem avaliação da sua qualidade) apresentou forte associação com o óbito perinatal após controle das demais variáveis, em conformidade com o que aponta a literatura $2,3,5,14$.

A variável instrução materna, utilizada como indicador da condição sócio-econômica e reconhecido fator de risco para mortalidade infantil e neonatal 6,32,33, não apresentou diferença entre casos e controles neste estudo, possivelmente por se tratar de população usuária do SUS, socialmente homogênea. Apesar de não detectada associação entre o tipo de parto e o óbito perinatal, a proporção de cesárea encontrada, ligeiramente acima do recomendado pela OMS 34, foi muito aquém dos valores médios do município para o mesmo ano, decorrente das elevadas taxas de parto operatório praticadas nos hospitais privados não conveniados ao SUS 29 .

O nascimento em horários com provável redução de equipe (feriado, final de semana e noite) não foi risco para o óbito perinatal neste estudo, como encontrado em estudos de países desenvolvidos, que tinham interesse em identificar aspectos da assistência que poderiam ser melhorados para a prevenção das mor- 
Modelo final da análise de múltipla regressão logística (OR ajustada) de variáveis de risco selecionadas para o óbito perinatal. Belo Horizonte, Minas Gerais, Brasil, janeiro-setembro de1999.

\begin{tabular}{|c|c|c|c|}
\hline Variável & $\begin{array}{l}\text { Partograma } \\
\text { OR* (IC95\%) }\end{array}$ & $\begin{array}{c}\text { Modelo } \\
\text { Avaliação fetal durante } \\
\text { trabalho de parto } \\
\text { OR* }^{*}(\mathrm{C} 95 \%)\end{array}$ & $\begin{array}{c}\text { Avaliação materna } \\
\text { durante trabalho de parto } \\
\text { OR* (IC95\%) }\end{array}$ \\
\hline \multicolumn{4}{|l|}{ Partograma } \\
\hline Sim & 1 & - & - \\
\hline Não & $2,76(1,21-6,26)$ & - & - \\
\hline \multicolumn{4}{|c|}{$\begin{array}{l}\text { Avaliação fetal durante } \\
\text { trabalho de parto (por hora) }\end{array}$} \\
\hline$\geq 1$ & - & 1 & - \\
\hline$<1$ & - & $1,75(0,61-5,02)$ & - \\
\hline \multicolumn{4}{|c|}{$\begin{array}{l}\text { Avaliação materna durante } \\
\text { trabalho de parto (por hora) }\end{array}$} \\
\hline$\geq 1$ & - & - & 1 \\
\hline$<1$ & - & - & $1,20(0,30-3,77)$ \\
\hline \multicolumn{4}{|c|}{ Categoria do hospital } \\
\hline Tipo 3 & 1 & 1 & 1 \\
\hline Tipo 2 & $0,60(0,13-2,63)$ & $0,78(0,19-3,15)$ & $0,76(0,19-2,99)$ \\
\hline Tipo 1 & $5,15(1,57-16,85)$ & $4,96(1,48-16,63)$ & $4,91(1,46-16,53)$ \\
\hline \multicolumn{4}{|c|}{ Idade materna (anos) } \\
\hline $20-34$ & 1 & 1 & 1 \\
\hline$<20$ & $1,13(0,45-2,81)$ & $1,26(0,52-3,06)$ & $1,14(0,47-2,73)$ \\
\hline$\geq 35$ & $2,76(0,85-8,89)$ & $3,46(1,14-10,53)$ & $3,37(1,10-10,31)$ \\
\hline \multicolumn{4}{|c|}{ Sexo do recém-nascido } \\
\hline Feminino & 1 & 1 & 1 \\
\hline Masculino & $2,44(0,99-5,26)$ & $2,44(1,075-5,55)$ & $2,56(1,15-5,88)$ \\
\hline \multicolumn{4}{|c|}{ Baixo peso ao nascer } \\
\hline Não & 1 & 1 & 1 \\
\hline Sim & $17,53(7,30-42,13)$ & $16,45(6,80-39,77)$ & $17,39(7,26-41,67)$ \\
\hline \multicolumn{4}{|l|}{ Pré-natal } \\
\hline Sim & 1 & 1 & 1 \\
\hline Não & $40,04(16,92-94,75)$ & $36,02(15,34-84,59)$ & $39,74(17,40-90,80)$ \\
\hline \multicolumn{4}{|c|}{ Doenças maiores na gravidez } \\
\hline Não & 1 & 1 & 1 \\
\hline Sim & $11,20(2,77-45,31)$ & $9,16(1,92-43,64)$ & $8,39(1,80-39,10)$ \\
\hline \multicolumn{4}{|c|}{ Doenças menores na gravidez } \\
\hline Não & 1 & 1 & 1 \\
\hline Sim & $13,86(4,45-43,09)$ & $11,63(3,98-34,02)$ & $11,53(4,01-33,10)$ \\
\hline \multicolumn{4}{|c|}{ Doenças do recém-nascido } \\
\hline Não & 1 & 1 & 1 \\
\hline Sim & $106,24(19,74-571,80)$ & $173,99(38,57-989,40)$ & $184,06(33,97-997,41)$ \\
\hline
\end{tabular}

* Odds ratio; excluídos registros ignorados. 
tes por asfixia 35,36. Por sua vez, a asfixia moderada (Apgar $<7$ no quinto minuto de vida), presente em $51 \%$ dos nascidos vivos que morreram no período perinatal, apresentou-se como fator de risco para o óbito perinatal e parece ser indicativo da gravidade da criança, assim como a presença de pediatra na sala de parto. Vale ressaltar que cerca de $25 \%$ dos nascimentos entre os controles não contaram com assistência de pediatra na sala de parto.

São poucos os estudos analíticos disponíveis no Brasil que abordam os aspectos da assistência ao parto e sua relação com a mortalidade perinatal, enfoque deste estudo. A maior parte dos estudos de avaliação da assistência obstétrica e neonatal é dirigida à assistência pré-natal ou à avaliação de estrutura dos hospitais e poucos tratam dos aspectos relativos ao processo de assistência. Por este motivo a discussão torna-se limitada a estudos internacionais, com realidades muito diferentes das do Brasil, como nos países desenvolvidos, onde predominam causas de mortalidade perinatal com menor possibilidade de prevenção 16 . Por sua vez, comparar o Brasil com países em desenvolvimento, onde predomina a assistência domiciliar ao parto, parece inapropriado.

Dentre os vários desafios identificados para a melhoria da assistência, destaca-se o acesso em tempo oportuno aos serviços de saúde, com garantia de acolhida imediata da gestante em trabalho de parto. Como demonstrado neste estudo, 8,4\% das gestantes cujos filhos morreram na fase perinatal foram admitidas em período expulsivo na maternidade, apontando possível dificuldade de acesso aos serviços de saúde, em contraste com o grupo controle. Em segundo lugar, além do acesso, deve ser assegurada a provisão da atenção e cuidado em saúde nos patamares reconhecidos de qualidade, o que nem sempre ocorre, como observado neste estudo, quando mulheres em trabalho de parto, já em assistência hospitalar, não receberam o cuidado requerido.

Orientações para o adequado acompanhamento do trabalho de parto estão disponíveis em diversas publicações oficiais, nacionais e internacionais $11,27,28,37,38,39$. A OMS recomenda a utilização do partograma - representação gráfica da curva de dilatação cervical durante o trabalho de parto - nas maternidades desde 199428,40 , considerando que facilita o acompanhamento do trabalho de parto e o diagnóstico precoce das distócias, indicando a necessidade oportuna de intervenção. A sua utilização indica claramente desvios no progresso do trabalho de parto, possibilitando intervenções antes que ocorram complicações 41 . No entanto, ape- sar de sua efetividade, o partograma não foi universalmente adotado, como demonstra estudo na Nigéria, onde apenas $25 \%$ dos profissionais utilizaram o partograma na sua prática cotidiana, apesar de $90 \%$ conhecerem o instrumento 42. Em maternidades do SUS em São Luís, Maranhão, apenas 39,1\% dos prontuários de gestantes em trabalho de parto possuíam o partograma e somente $13,3 \%$ destes estavam preenchidos 43 . No presente estudo a não utilização do partograma aumentou de maneira importante o risco de óbito perinatal, independentemente da categoria do hospital e de outras variáveis (OR = 2,76; IC95\%: 1,22-26; $\mathrm{p}=0,015)$.

Recomenda-se que o acompanhamento do trabalho de parto e do feto seja realizada inicialmente a cada sessenta minutos e a seguir a cada trinta minutos, conforme progrida a fase ativa do trabalho de parto 27,28 . O critério adotado neste estudo, de pelo menos uma avaliação por hora, baseou-se no que poderíamos chamar de critério mínimo de qualidade para assistência à gestante em trabalho de parto em maternidades. As gestantes neste estudo foram admitidas em trabalho de parto, permaneceram internadas por no mínimo uma hora, em média por 6,3 horas, e $50 \%$ por quatro horas. Ainda assim, mais de um terço das gestantes e fetos tiveram apenas uma avaliação durante o trabalho de parto. Este elevado porcentual de avaliação materna e fetal inadequada durante o trabalho de parto é um indicador da qualidade da assistência, ainda que avaliado de forma indireta, por se basear em registros de prontuários. Quando a avaliação fetal foi inadequada durante o trabalho de parto houve maior risco de morte perinatal, porém a associação não permaneceu significativa no modelo ajustado para as demais variáveis. Estudo realizado no Rio de Janeiro encontrou 47,5\% de registros incompletos de batimento cardíaco fetal durante o acompanhamento do trabalho de parto 44 . Também em Teresina, Piauí, foram observados indicadores insatisfatórios em relação à aferição de pressão arterial e ausculta de batimentos cardíacos fetais no pré-parto 45 . Neste estudo não foi encontrada associação estatística entre a avaliação materna durante o trabalho de parto e o óbito perinatal. Isto pode ser explicado pelo percentual semelhante (e elevado) de casos $(83,9 \%)$ e controles $(78,7 \%)$ com avaliação materna inadequada, resultando em ausência de associação estatística com o óbito perinatal.

Em relação à categoria do hospital, o hospital tipo 1 apresentou-se como risco para o óbito perinatal em todos os modelos. Estes hospitais-maternidades receberam escore baixo na 
avaliação de estrutura realizada por Costa et al. 21 e não dispunham de condições para o atendimento adequado das complicações que ocorrem durante o nascimento, esperadas em cerca de 10 a $15 \%$ dos partos, mesmo para as gestações de risco habitual 11. Nesses hospitais, $38,1 \%$ dos nascidos vivos não receberam assistência de pediatra na sala de parto, em contraste com $2,5 \%$ e $2,2 \%$ nos hospitais tipo 2 e tipo 3 , respectivamente.

A rede assistencial para gestante e recémnascido, além de integrada, hierarquizada e regionalizada, de forma a dar acesso à gestante em tempo oportuno no momento do parto, deve garantir também que todos os estabelecimentos de saúde onde se realizam partos sejam estruturados para o atendimento resolutivo das complicações que podem ocorrer no nascimento - situações esperadas mas não previsíveis - disponibilizando equipamentos, insumos e equipe capacitada para prestar o primeiro atendimento adequado às urgências maternas e neonatais 46,47,48. Destacamos que a gestão do SUS em Belo Horizonte, a partir da avaliação das maternidades realizada em 1999, executou uma série de ações para organização e qualificação da assistência perinatal, excluindo cinco maternidades com baixo escore de qualidade do atendimento pelo SUS e apoiando a reestruturação das demais, além da ampliação de leitos para recém-nascido de alto risco, capacitação dos profissionais e vinculação das gestantes às maternidades para melhorar o acesso à assistência no momento do parto 49 .

Não foi observada a associação entre o hospital tipo 2 e o óbito perinatal. Por um lado, a amostra do estudo pode ter sido insuficiente para detectar associação significativa, ou ainda, o estudo pode não ter considerado outras possíveis variáveis de confusão. Por outro lado, pode de fato não existir diferença entre o hospital tipo 2 e o hospital tipo 3 em relação ao risco de morte perinatal. As unidades de cuidados intermediários hospital tipo 2 em Belo Horizonte, denominadas "unidade neonatal de cuidados progressivos”, executam todas as ações de assistência que o recém-nascido demanda, inclusive cuidado intensivo, sem necessidade de sua transferência para uma UTI 29. Considerandose este aspecto, seria de grande importância aprofundar esta análise com a realização de outros estudos, que poderiam subsidiar o planejamento das ações de saúde para a redução da mortalidade perinatal no país, por exemplo, com ampliação de unidades regionalizadas de cuidados intermediários, e não necessariamente UTIs, para o alcance de resultados favoráveis para a gestante e para o recém-nascido.
O baixo peso ao nascer manteve-se associado ao óbito perinatal após o controle de variáveis de confusão em todos os modelos, reforçando o peso ao nascer como fator determinante da sobrevivência infantil. Importante ressaltar que uma grande parcela dos óbitos $(37,9 \%)$ ocorreu em crianças com peso acima de $2.500 \mathrm{~g}$, demonstrando o potencial de prevenção destas mortes, que devem ser considerados eventos-sentinela. A associação entre a morte perinatal e doenças na gravidez (como a hipertensão, DHEG, diabetes, hemorragia e mesmo doenças consideradas de menor importância), amplamente documentada na literatura, foi também observada neste estudo 14 . Da mesma forma, as doenças do recém-nascido persistiram associadas ao óbito perinatal nos modelos de regressão, apesar da pouca precisão das estimativas, decorrente do pequeno número de crianças com estas condições (17 no total e apenas 1 entre os controles). A idade materna não foi significativa no modelo partograma, possivelmente por serem variáveis correlacionadas, tendo as gestantes com mais de 35 anos apresentado maior chance de serem acompanhadas com partograma durante o trabalho de parto.

Um número muito pequeno de gestantes em trabalho de parto prematuro recebeu corticosteróide, $6,7 \%$ daquelas com idade gestacional entre 28 e 34 semanas, indicando dificuldade de acesso das gestantes e crianças a este tipo de benefício. Da mesma forma, poucas crianças nascidas vivas parecem ter tido acesso a cuidados mais apropriados à sua grave condição - já que morreram no período perinatal - pois cerca de $40 \%$ permaneceram em assistência em unidades de baixa complexidade (alojamento conjunto ou berçário comum) e poucas crianças $(5,8 \%)$ receberam cuidados intensivos e conseqüentemente, acesso a medicamentos eficazes para prematuros, como o surfactante.

Sobre a asfixia ao nascer, a OMS estima que esta seja a causa direta de $21 \%$ das mortes neonatais e responsável por $8 \%$ das mortes infantis no mundo 50; estudo realizado no Brasil apontou que a hipóxia ou anóxia representaram 11,7\% dos óbitos ocorridos em 1985 2,48. Alguns trabalhos nacionais destacam as mortes por asfixia no contexto das taxas elevadas de mortalidade perinatal e neonatal por causas consideradas evitáveis: concentram-se em crianças com peso adequado ao nascer e apresentam grande potencial de redução pelo adequado acompanhamento do trabalho de parto 1,7 , 10,15,42,51. Diversos autores apontam a asfixia como a principal causa de natimortalidade, principalmente em crianças no final da gestação, 
52,53,54 e o declínio da taxa de mortalidade fetal nos países desenvolvidos foi atribuída ao monitoramento e melhoria na assistência durante o trabalho de parto 14. Esse mesmo autor conclui que a identificação da gestante sem assistência pré-natal, a implementação da identificação de risco na gravidez, o diagnóstico e manejo do crescimento fetal intra-uterino e das condições maternas, mas especialmente o foco no manejo das complicações durante o trabalho de parto, podem reduzir a mortalidade fetal. Na medida em que a assistência ao trabalho de parto melhora, a asfixia neste momento diminui, e como conseqüência, apenas 10,0 a $15,0 \%$ das mortes fetais em países desenvolvidos ocorrem intraparto. As mortes intraparto freqüentemente envolvem manejo inapropriado do parto e nascimento, ocorrem em sua maioria em gestações de baixo risco e são mais facilmente preveníveis do que os óbitos anteparto. Portanto, a maior influência do serviço de saúde para a redução da mortalidade fetal ocorre durante o trabalho de parto e nascimento. No entanto, a importância clínica e epidemiológica dos óbitos fetais ainda não foi suficientemente reconhecida pelos serviços de saúde no Brasil.

A qualidade da assistência precisa ser amplamente discutida e repensada no cotidiano do trabalho em saúde. As questões relativas à estrutura dos serviços são de fundamental importância, mas não garantem que o processo de trabalho seja desempenhado de maneira adequada e este é, de forma geral, um aspecto pouco avaliado. As intervenções mais efetivas podem ser aquelas relativas às alterações dos procedimentos relacionados com o trabalho de rotina nos serviços, o trabalho integrado entre os profissionais e a relação com o usuário, e não necessariamente maiores gastos com tecnologia médica complexa 13,55. No caso dos óbitos perinatais por causas evitáveis, o cuidado em saúde destinado a um grupo de condições como a asfixia intraparto pode responder por muito do excesso da morbidade e mortalidade perinatal, principalmente em crianças com peso adequado ao nascer. Muitas vezes procedimentos simples, como o monitoramento efetivo do processo do parto e nascimento, que podem ser extremamente eficazes, são negligenciados pelos profissionais 42 . Grande parte dos problemas relaciona-se com o cuidado cotidiano interpessoal, fundamental no acompanhamento do trabalho de parto, para a prevenção da mortalidade e morbidade associadas às complicações no momento do nascimento, como a obstrução do trabalho de parto ou o trabalho de parto prolongado.
É reconhecida a profunda influência dos fatores sócio-econômicos e da organização da rede assistencial de saúde na mortalidade perinatal. Mas é preciso ainda reconhecer e superar o diferencial no cuidado de saúde que existe entre a população pobre, neste estudo representada pela população usuária do SUS, e a de nível sócio-econômico melhor. Como ressalta Victora et al. 33, os serviços de saúde que atendem às comunidades pobres são geralmente de pior qualidade, menos organizados, com profissionais de saúde pouco sensíveis às suas necessidades, situação que demanda esforços específicos para se prover o melhor cuidado para quem tem maior necessidade.

Como uma limitação desta pesquisa, apontamos o fato de se basear em dados de prontuários hospitalares, sujeitos a problemas de qualidade e completude de informações. Restringe-se ainda à metodologia quantitativa e outros enfoques da avaliação da qualidade da atenção em saúde, como os estudos qualitativos da atenção e de satisfação das usuárias, que poderiam complementar esta análise.

\section{Conclusão}

Para a redução da mortalidade infantil e perinatal no Brasil os esforços em ações de saúde são de fundamental importância, particularmente a melhoria do acesso e qualidade da atenção pré-natal e atenção ao parto e nascimento. Os resultados deste estudo apontam a necessidade da adoção de medidas básicas e efetivas de qualificação da assistência à gestante e ao recém-nascido, como a estruturação dos hospitais e o adequado monitoramento do trabalho de parto, de modo a oferecer segurança no atendimento ao parto, propiciando o nascimento seguro e a redução da mortalidade perinatal por causas evitáveis. É preciso repensar o papel da atenção pré-parto, muitas vezes relegada a um segundo plano no dia-a-dia do trabalho dos hospitais e maternidades. Além de estrutura adequada, as maternidades devem contar com equipe treinada, e mais que tudo, disponível para prestar o cuidado necessário, humanizado e em tempo oportuno, para a redução de uma das causas de morte perinatal com maior potencial de prevenção por cuidados de saúde: a asfixia intraparto.

De forma geral, o período crucial entre o final da gravidez, o trabalho de parto e o momento do nascimento não receberam ainda a atenção merecida do sistema de saúde. Neste momento o risco de resultados adversos para a mulher e a criança é muito grande, e os fatores 
de risco mais significativos para a morte fetal e neonatal também afetam profundamente a mortalidade materna, ainda muito elevada no país. Ganhos mútuos para a saúde materna e perinatal poderão ser alcançados, com redução significativa dessas mortes precoces, se as complicações do parto e nascimento forem antecipadas, reconhecidas e imediatamente atendidas, constituindo-se ações de saúde prioritárias para o país.

\section{Resumo}

Este trabalho analisa a associação entre a morte perinatal e o processo de assistência hospitalar ao parto, considerando-se que grande parte das mortes perinatais pode ser prevenível pela atenção qualificada de saúde e que a avaliação da qualidade da assistência perinatal ao parto é necessária para a redução da morbi-mortalidade perinatal. Realizou-se estudo caso-controle de base populacional dos óbitos perinatais $(n=118)$ e nascimentos $(n=492)$, ocorridos em mater nidades do Sistema Único de Saúde (SUS) de Belo Horizonte, Minas Gerais, Brasil. Sexo masculino, prematuridade, doenças na gravidez, baixo peso ao nascer, doenças do recém-nascido, não realização de pré-natal, não utilização de partograma e menos de uma avaliação fetal por hora durante o trabalho de parto apresentaram associação estatisticamente significativa com o óbito perinatal. No modelo de regressão logística múltipla, não utilização do partograma durante o trabalho de parto e tipo de maternidade apresentaram-se como fatores de risco independentes para a morte perinatal. O estudo indica que é deficiente a qualidade da assistência hospitalar ao parto e que aspectos da estrutura dos serviços e do processo de assistência relacionam-se com a mortalidade perinatal por causas evitáveis.

Avaliação dos Serviços; Cuidado Pré-Natal; Mortalidade Infantil

\section{Colaboradores}

S. Lansky participou de todas as etapas do estudo e da elaboração do artigo, sendo responsável pela versão final. C. C. César participou do planejamento do estudo, análise dos resultados, orientação estatística e revisão do artigo. M. C. Leal contribuiu no planejamento do estudo, discussão dos resultados e redação do artigo. L. C. Monteiro Neto participou da construção e processamento do banco de dados e análise dos resultados. E. França foi responsável pela orientação geral, participando de todas as etapas do trabalho.

\section{Agradecimentos}

Trabalho financiado pela Organização Pan-Americana da Saúde/Organização Mundial da Saúde (AMR/ 99/078643-01) e pelo Conselho Nacional de Desenvolvimento Científico e Tecnológico. 


\section{Referências}

1. Lansky S, França E, Leal MC. Mortes perinatais evitáveis em Belo Horizonte, Minas Gerais, Brasil, 1999. Cad Saúde Pública 2002; 18:1389-400.

2. Victora CG. Intervenções para reduzir a mortalidade infantil. Rev Bras Epidemiol 2001; 4:3-69.

3. Victora CG, Barros FC. Infant mortality due to perinatal causes in Brazil: trends, regional patterns and possible interventions. São Paulo Med J 2001; 119:33-42.

4. Lansky S, França E, Leal MC. Mortalidade perinatal e evitabilidade: revisão da literatura. Rev Saúde Pública 2002; 36:759-72.

5. Leal MC, Gama SGN, Campos M, Cavalini LT, Garbayo LS, Brasil CL, et al. Fatores associados à morbi-mortalidade perinatal em uma amostra de maternidades públicas e privadas do Município do Rio de Janeiro. Cad Saúde Pública 2004; 20 Suppl 1:S20-33.

6. Almeida MF, Novaes HMD, Alencar GP, Rodrigues LR. Mortalidade neonatal no Município de São Paulo: influência do peso ao nascer e de fatores sócio-demográficos e assistenciais. Rev Bras Epidemiol 2002; 5:93-107.

7. Barros FC, Victora CG, Vaughan JP. Causas de mortalidade perinatal em Pelotas, RS (Brasil): utilização de uma classificação simplificada. Rev Saúde Pública 1987; 21:310-6.

8. Bartlett A, Bocaletti MEP, Bocaletti MA. Reducing perinatal mortality in developing countries: high risk or improved labour management? Health Policy Plan 1993; 8:360-8.

9. Carvalho ML. Mortalidade neonatal e aspectos da qualidade da atenção à saúde na região metropolitana do Rio de Janeiro [Dissertação Mestrado]. Rio de Janeiro: Escola Nacional de Saúde Publica, Fundação Oswaldo Cruz; 1993.

10. Leite AJM, Marcopito LF, Diniz RLP, Silva AVS, Souza LCB, Borges JC, et al. Mortes perinatais no Município de Fortaleza, Ceará: o quanto é possível evitar? J Pediatr (Rio de J) 1997; 73:388-94.

11. Bale JR, Stoll J, Lucas AO, editors. Improving birth outcomes-meeting the challenge in the developing world. Washington DC: The National Academies Press; 2003.

12. Rutstein DD, Berenberg W, Chalmers T, Child CG, Fishman AP, Perrin EB. Measuring the quality of medical care: a clinical method. N Engl J Med 1976; 294:582-8.

13. Wigglesworth JS. Monitoring perinatal mortality -a pathophysiological approach. Lancet 1980; 27:684-6.

14. Conde-Agudelo A, Belizan JL, Dias-Rosselo JL. Epidemiology of fetal death in Latin American. Acta Obstet Gynecol Scand 2000; 79:371-8.

15. Menezes AMB, Barros FC, Victora CG, Alves C, Rocha C, Albernaz E, et al. Mortalidade perinatal em duas coortes de base populacional no Sul do Brasil: tendências e diferenciais. Cad Saúde Pública 1996; 12 Suppl 1:33-41.

16. Finan A, Clarke A, Matthews TG, Ledwidge M, Gillan J, Barry-Kinsella C, et al. Strategies for reduction of neonatal mortality. Ir J Med Sci 1999; 168:265-7.

17. Sociedade Civil Bem-estar Familiar no Brasil. Pesquisa Nacional sobre Demografia e Saúde, 1996.
Rio de Janeiro: Sociedade Civil Bem-estar Familiar no Brasil/Demographic and Health Survey; 1997.

18. Lisboa AMJ. Situação atual da perinatologia no Brasil. In: Fontes JAS, organizador. Perinatologia social. São Paulo: Editora Byk Procienx; 1984. p. 88-94.

19. Ojeda NS. Evaluación de servicios de salud materno-infantil en América Latina. Foro Mund Salud 1992; 13:140-3.

20. Accioly MC. Determinantes da mortalidade neonatal em Belo Horizonte (1993): subsídios para o planejamento da atenção materno-infantil [Dissertação Mestrado]. Belo Horizonte: Universidade Federal de Minas Gerais; 1997.

21. Costa JO, Xavier CC, Proietti FA, Delgado MS Avaliação dos recursos hospitalares para assistência perinatal em Belo Horizonte, Minas Gerais. Rev Saúde Pública 2004; 38:701-8.

22. Donabedian A. The quality of care. How can it be assessed? JAMA 1988; 260:1743-8.

23. Leatherman S, McCarthy D. Quality of care for children and adolescents. Chapel Hill: The University of North Carolina at Chapel Hill; 2004.

24. Evangelista PA, Lansky S, Rodrigues C, Porto YF, Araripe MG, Faria R, et al. Avaliação da Qualidade de Assistência em Maternidades do SUS-BH, 1999. Rev Assoc Méd Minas Gerais 2002; 12:66.

25. Organização Mundial da Saúde. Classificação Internacional de Doenças. 10a Revisão. São Paulo: Organização Mundial da Saúde; 1994.

26. Friche AA. Qualidade do sistema de informação sobre nascidos vivos em maternidades do SUSBH, 1999 [Dissertação de Mestrado]. Belo Horizonte: Universidade Federal de Minas Gerais; 2004.

27. Organização Mundial da Saúde. Maternidade segura. Assistência ao parto normal: um guia prático. Genebra: Organização Mundial da Saúde; 1996.

28. Ministério da Saúde. Parto, aborto e puerpério. Assistência humanizada à mulher. Brasília: Ministério da Saúde; 2001.

29. Secretaria Municipal de Saúde de Belo Horizonte. Relatório da Comissão Perinatal 1999. Belo Horizonte: Secretaria Municipal de Saúde de Belo Horizonte; 1999.

30. Wigglesworth JS. Classification of perinatal deaths. Soz Praventivmed 1994; 39:11-4.

31. Keeling JW, MacGillivray I, Golding J, Wigglesworth J, Berry J, Dunn PM. Classification of perinatal death. Arch Dis Chil 1989; 64:1345-51.

32. França E, Souza JM, Guimarães MDC, Goulart EM, Colosimo E, Antunes CMF. Associação entre fatores sócio-econômicos e mortalidade infantil por diarréia, pneumonia e desnutrição em região metropolitana do Sudeste do Brasil: um estudo caso-controle. Cad Saúde Pública 2001; 17:143747.

33. Victora CG, Wagstaff A, Schellenberg JA, Gwatkin D, Claeson M, Habicht J. Applying an equity lens to child health and mortality: more of the same is not enough. Lancet 2003; 61:233-41.

34. World Health Organization. Appropriate technology for birth. Lancet 1985; 2:436-7. 
35. Chalmers JW, Shancks E, Paterson S, Mcinneny K, Baird D, Penney G. Scottish data on intrapartum related deaths are in same direction as Welsh data. BMJ 1998; 317:539-40.

36. Stewart JH, Andrews J, Cartlidge PHT. Numbers of deaths related to intrapartum asphyxia and timing of birth in all Wales perinatal survey, 199395. BMJ 1998; 316:657-60.

37. Ministério da Saúde. Assistência institucional ao parto, ao puerpério e ao recém nascido. Brasília: Ministério da Saúde; 1989.

38. Ministério da Saúde. Programa de assistência integral à saúde da mulher. Assistência institucional ao parto, ao puerpério e ao recém-nascido. Brasília: Ministério da Saúde; 1991.

39. World Health Organization. Mother-baby package: implementing safe motherhood in countries. Geneva: World Health Organization; 1998.

40. World Health Organization. World Health Organization partograph in the management of labour. Lancet 1994; 343:1399-404.

41. Buchmann EJ, Gulmesoglu AM, Nikodem VC. Partogram for assessing progress of labour (Cochrane Review). In: The Cochrane Library, Issue 2, 2002. Oxford: Update Software.

42. Umezulike AC, Onah HE, Okaro JM. Use of the partograph among medical personnel in Enugu, Nigeria. Int J Gynaecol Obstet 1999; 65:203-5.

43. Alves MTS, Silva AAM. Avaliação da qualidade de maternidades-assistência à mulher e ao recémnascido no Sistema Único de Saúde. São Luís: Universidade Federal do Maranhão/Fundo das Nações Unidas para a Infância; 2000.

44. Garbayo LS, Campos MR, Leal MC, Cunha CB. Maternidades públicas e conveniadas ao SUS: avaliação da qualidade de assistência ao pré-parto no Município do Rio de Janeiro, 1999-2000. Rev Bras Epidemiol 2002; Num Esp:347.
45. Borba AS, Brandim MRR, Nogueira NN. Análise situacional da assistência obstétrica e perinatal em maternidades de Teresina. Teresina: Fundo das Nações Unidas para a Infância; 2001.

46. Salinas AM, Coria I, Reyes H, Zambrana M. Effect of quality of care on preventable perinatal mortality. Int J Qual Health Care 1997; 9:93-9.

47. Leal MC, Viacava F. Maternidades do Brasil. Radis 2002; (2):8-26.

48. World Health Organization. Managing complications in pregnancy and childbirth. A guide for midwives and doctors. Geneva: World Health Organization; 2003.

49. Lansky S. BH Viva Criança: acelerando a redução da mortalidade infantil. Saúde Digital 2002; 10. http://www.pbh.gov.br/smsa/saudedigital/outubro2002/bhvivacrianca.html.

50. Bryce J, Boschi-Pinto C, Shibuya K, Black RE. WHO estimates of the causes of death in children. Lancet 2005; 365:1147-52.

51. Ministério da Saúde/Fundo das Nações Unidas para a Infância. A mortalidade perinatal e neonatal no Brasil. Brasília: Ministério da Saúde/Fundo das Nações Unidas para a Infância; 1998.

52. Ribeiro VS, Silva AAM. Tendências da mortalidade neonatal em São Luís, Maranhão, Brasil, 1979 a 1996. Cad Saúde Pública 2000; 16:429-38.

53. Ashley D, Maccaw-Binns A, Golding J, Keeling J, Ecoffery C, Coard K, et al. Perinatal mortality survey, 1994. Paediatr Perinat Epidemiol 1994; 8:6-16.

54. Raghuveer G. Perinatal deaths: relevance of Wigglesworth classification. Paediatr Perinat Epidemiol 1992; 6:45-50.

55. Merhy EE. Saúde: a cartografia do trabalho vivo. São Paulo: Editora Hucitec; 2002.

Recebido em 21/Out/2004

Versão final reapresentada em 03/Mai/2005

Aprovado em 24/Jun/2005 